\title{
Molecular characterization and testing in acute myeloid leukemia
}

\author{
Gerald B. W. Wertheim ${ }^{1}$
}

Received: 22 August 2014 / Accepted: 9 March 2015 / Published online: 14 April 2015

(C) Springer-Verlag Berlin Heidelberg 2015

\begin{abstract}
Acute myeloid leukemia (AML) is a clinically and biologically heterogeneous group of neoplasms found in both the adult and pediatric populations. Many of the mutations that underlie AML pathogenesis have been elucidated and include both large-scale genomic events such as chromosomal additions, deletions, and translocations, as well as small-scale point mutations in tumorigenic genes. As patient prognosis and therapeutic decisions are largely determined by these genetic events, multifaceted genetic analysis of tissue sample from AML patients is required for proper diagnosis. Thus, an understanding of both the genetic events associated with AML and the testing modalities that assess these aberrations is essential for appropriate sample evaluation. Additionally, recognition of the molecular differences between adult and pediatric AML may provide insights into critical pathways involved in AML pathogenesis.
\end{abstract}

Keywords Acute myeloid leukemia $\cdot$ Molecular alterations . Testing modalities $\cdot$ Genetic events

Acute myeloid leukemia (AML) affects $\sim 12,000$ new patients in the USA each year [1]. Although this leukemia mainly occurs in older adults, a significant proportion of patients are infants and children, and roughly 800 pediatric cases of AML - accounting for approximately $20 \%$ of pediatric leukemia - are diagnosed yearly in the USA [2-4].

Gerald B. W. Wertheim

wertheimg@email.chop.edu

1 Department of Pathology and Laboratory Medicine, Children's Hospital of Philadelphia and the Perelman School of Medicine at the University of Pennsylvania, Philadelphia, PA 19104, USA
Both adult and pediatric AML are characterized by an overproduction of myeloid progenitor cells that are unable to develop into mature nonlymphoid hematopoietic elements $[5$, 6]. This typically (but not always) manifests as an overabundance of blasts that account for $>20 \%$ of total nucleated cells. The rather broad definition of AML clearly encompasses a wide variety of discrete pathologic entities that display highly variable phenotypic features. For instance, there is a spectrum of morphologic and immunophenotypic findings in AML, with tumor cells ranging from very immature $\mathrm{CD} 34+$ blasts to cells showing granulocytic, monocytic, erythroid, or megakaryocytic lineage commitment $[6,7]$. Likewise, patients with AML show a wide of clinical outcomes, with some responding quite well to conventional chemotherapy and experiencing sustained remissions, while others require bone marrow transplantation for long-term survival, and still others display refractory disease with rapid demise [8-10].

The pathologic and clinical phenotypes of AML are driven, in large part, by the specific genotypic alterations harbored by the tumor cells. Since the unifying features of AML are increased proliferation of hematopoietic elements and the inability of these cells to differentiate, Gilliland and coworkers categorized mutations in AML as class I (those that are related to increased proliferation) and class II (those that are related to lack of maturation), and they surmised that these two classes of mutations must both occur to convert a normal hematopoietic cell into one giving rise to AML [11,12]. Recently, a third class of mutations (class III), involving genes that regulate epigenetic modifications and chromatin structure, has also emerged as critical for AML pathogenesis [13]. Although these three types of mutations are not always identified in a single tumor, many mutations recurrently found in AML fall into one of these three categories. Additionally, this three-class model 
of mutations is clearly not comprehensive, as large-scale sequencing studies have revealed mutations in other genes, such as splicing factors, that influence additional cellular properties [14].

Though these types of AML mutations underlie the general similarities among AML cases, the wide diversity of AML phenotypes suggests a similarly wide diversity of AMLassociated mutations. Indeed, numerous recurrent driver mutations are found among AML patients, and not surprisingly, many of these mutations correlate with specific phenotypic features, including cytomorphology, immunophenotype, and clinical outcomes [5, 14-16]. The importance of the underlying mutational events driving both AML biology and the clinical course of patients has been codified in the WHO classification of hematopoietic tumors, and current clinical workups for proper AML categorization are largely dependent on multiple genetic tests of blood or bone marrow samples [6, 17]. In addition to the mutations formally used for AML subclassification, many novel AML driver mutations have recently been identified through large-scale genome sequencing projects $[14,18,19]$. As many of these mutations have prognostic implications, genetic testing at these additional will likely become standard in the diagnostic workup of both adult and pediatric AML.

This article describes many of the mutations found in AML, with particular attention paid to those currently used to define AML subclasses, as well as to those with prognostic significance in pediatric patients. Since identifying these mutations in AML requires multiple assays to be performed on blood, bone marrow, or other tissue specimens, a basic understanding of testing methodologies is essential for proper specimen triage. Thus, discussions of both standard and emerging clinical molecular assays are included. Finally, important molecular distinctions between pediatric and adult AML will be highlighted along with possible implications of these differences.

\section{Karytopic ("large-scale") abnormalities in AML}

Since the original method of examining the genome of normal and leukemic cells was by microscopic analysis of metaphase spreads, the earliest identified and most well-studied genetic abnormalities associated with AML are those involving whole chromosomes or large parts of chromosomes [10]. These abnormalities include numerical abnormalities (i.e., chromosomal gains and losses), as well as translocations and inversions. Many of these events not only correlate with the morphology or immunophenotype of the leukemic cells but also define patient prognosis and guide antileukemic therapy [5, $10,20]$. Due to the primary importance of karyotypic abnormalities in AML, the WHO classifies seven AML subtypes based on a defining chromosomal defect [6]. Furthermore, many of the numerical chromosomal abnormalities found in AML are important diagnostic findings for an additional WHO-defined subcategory of AML (i.e., AML with myelodysplasia-related changes).

Three of the translocations that define AML subgroups are associated with relatively favorable patient outcomes [10,15]. These are $\mathrm{t}(15 ; 17)(\mathrm{q} 24 ; \mathrm{q} 21), \mathrm{t}(8 ; 21)(\mathrm{q} 22 ; \mathrm{q} 22.3)$, and $\operatorname{inv}(16)(\mathrm{p} 13.1 \mathrm{q} 22)$ or $\mathrm{t}(16 ; 16)(\mathrm{p} 13.1 ; \mathrm{q} 22)$. The resulting fusions disrupt transcription factors essential for myeloid differentiation.

The $\mathrm{t}(15 ; 17)$ subtype occurs in $\sim 10 \%$ pediatric AML and essentially has a 1:1 correlation with acute promyelocytic leukemia (APL), also known as AML-M3 in the now obsolete FAB classification. The translocation fuses the alpha receptor for retinoic acid (RARA) on chromosome 17 to the inducer of promyelocytic leukemia $(P M L)$ locus on chromosome 15 . RARA is a critical transcriptional regulator of myeloid differentiation, and the fusion creates a dominant negative form of the protein, leading to a differentiation block at the promyelocyte stage [21, 22]. Rapid recognition of this entity, cytologically characterized by an abundance of atypical promyelocytes with bilobed nuclei, and molecular confirmation of the translocation is essential for appropriate clinical management since patients with this AML subtype are at high risk for disseminated intravascular coagulation and devastating vascular events [5]. Treatment for patients with $\mathrm{t}(15 ; 17)$ is unique in that pharmacologic dosing of all-trans retinoic (ATRA) is the mainstay of therapy [23]. Binding of ATRA to the PML-RARA fusion relieves the dominant negative inhibition of the fusion protein and leads its degradation by ubiquitin-mediated proteolysis. The use of ATRA in APL has completely changed the prognosis for patients with AML harboring the PML-RARA fusion. Prior to its use, AML with $\mathrm{t}(15 ; 17)$ had a dismal prognosis, whereas current long-term remission rates exceed $90 \%$ [24].

Almost all patients with APL morphology are positive for the PML-RARA fusion, yet there are variant RARA translocation partners that can lead to APL-like cytology. These include fusions with nucleophosmin (NPM1) [25], nuclear mitotic apparatus protein (NUMA1) [26], zinc finger and BTB-domain containing protein 16 (ZBTB16) [27, 28], and signal transducer and activator of transcription 5b (STAT5B) [29]. Although rare, these variants are important to recognize since a subset does not respond to ATRA therapy and alternative therapeutic strategies must be employed.

The other two translocations associated with a relatively favorable prognosis in both adults and children are $\mathrm{t}(8 ; 21)$ and inv(16)/t(16;16) [3, 24, 30-32]. The former chromosomal defect, found in $10-15 \%$ of pediatric AML, fuses the runtrelated transcription factor 1(RUNX1, formerly known as the core binding factor subunit alpha 2 ) to its translocation partner, $R U N X 1 T 1$, on chromosome 8 . The latter inversion/translocation, found in $\sim 5 \%$ of adult and pediatric AML, fuses the 
beta subunit of the core binding factor $(C B F B)$ to the heavy chain of myosin 11 (MYH11) [33]. Both of these genetically defined subgroups have cytologic and immunophenotypic correlates, though they are not as strong as that between APL and $t(15 ; 17)$. Blasts with $t(8 ; 21)$ have characteristic salmon-pink cytoplasmic inclusions, thin Auer rods with tapered ends, and basophilic cytoplasmic rims and may express lymphoid-associated proteins, especially CD19 [5, 34]. These blasts are typically found in a background of myeloid maturation (i.e., FAB-M2). Additionally, there is a recognized correlation between $\mathrm{t}(8 ; 21)$ and the presence of granulocytic sarcomas, and this correlation seems particularly strong in the pediatric population $[35,36]$. The subgroup of AML with $\operatorname{inv}(16) / t(16 ; 16)$ shows significant morphologic overlap with the FAB entity AML-M4 Eo, characterized by myelomonocytic differentiation and numerous immature cells with eosinophilic and basophilic granules [37, 38]. Although both RUNX1-RUNX1T1 and CBFB-MYH11 AML subclasses (collectively known as core-binding factor AML) are associated with a favorable prognosis, additional mutations can modify tumor aggressiveness. One such modifying event is an activating mutation in the v-kit Hardy-Zuckerman 4 feline sarcoma viral oncogene homolog (KIT), which downgrades the prognosis of core-binding factor leukemias from good to intermediate [39]. This effect of KIT on prognosis, however, seems to be more profound in adult than in pediatric AML [40].

On the other end of the prognostic spectrum from APL and core-binding factor AML are AML with $\mathrm{t}(6 ; 9)(\mathrm{p} 23 ; \mathrm{q} 34)$, fusing the $D E K$ proto-oncogene to a nucleoporin gene (NUP 214), and AML with inv(3)(q21q26.2) or $\mathrm{t}(3 ; 3)(\mathrm{q} 21 ; \mathrm{q} 26.2)$ which juxtaposes ribophorin $1(R P N 1)$ to the MDS1 and EVI1 complex locus (MECOM) [24, 30, 41]. Both of these entities occur infrequently in the pediatric population, yet like the more common translocations described above, these, too, correlate with distinct cytologic features. AML with DEK-NUP214 is associated with myelodysplasia in virtually all cases and frequently shows basophilia [42]. AML with inv(3)/t(3;3) also shows myelodysplasia with atypical megakaryocytes and giant platelets [43]. Interestingly, the clinical phenotype of this latter leukemia is likely related to overexpression of $M E C O M$, as the translocation brings a GATA2 enhancer element in proximity to the MECOM locus $[44,45]$. As expected, both inv(3)/t(3;3) AML and AML with increased MECOM expression in the absence of the chromosomal rearrangements have dismal prognoses [46-49]. Since $\mathrm{t}(6 ; 9)$ and $\operatorname{inv}(3) / \mathrm{t}(3 ; 3)$ AML are rare, they are not specifically targeted in routine diagnostic workups for pediatric AML; however, it is important to test for these alterations in cases with characteristic cytology given the association with aggressive disease.

At least three translocations are important for pediatric patients with AML displaying megakaryoblastic differentiation
(AMKL). One translocation, $\mathrm{t}(1 ; 22)(\mathrm{p} 13 ; \mathrm{q} 13)$, fuses RNAbinding motif protein 15 (RBM15) to megakaryoblastic leukemia (translocation) $1(M K L 1)$ and defines a WHO-described subclass of AML [6]. This translocation is typically seen in infant AMKL, and patients with this somatic translocation exhibit a more favorable clinical course than those who lack it. Two other AMKL-associated chromosomal rearrangements are not formally incorporated into the WHO classification. These are inv(16)(p13.3q24.3) which encodes a fusion between a core-binding factor gene (CBFA2T3) to a GLIS family zinc finger gene (GLIS2) and $\mathrm{t}(11 ; 12)(\mathrm{p} 15 ; \mathrm{q} 35)$ encoding a fusion between a nucleoporin protein and lysine-specific histone methylase (NUP98-KDM5a) [50, 51]. These two translocations account for $\sim 25$ and $\sim 10 \%$ of pediatric AMKL cases, respectively. It should be noted that the CBFA2T3GLIS2 translocation is not restricted to AMKL, and one study has shown that half of the AML cases with this fusion does not show megakaryocytic differentiation [52].

Perhaps the most interesting locus involved in AMLassociated translocations is the mixed-lineage leukemia (MLL) gene, now also known as lysine methyltransferase 2A $(K M T 2 A)$ located at 11q23. MLL translocations are found in both myeloid and lymphoid acute leukemia [3]. For AML, 11 q23 translocations are frequently (but certainly not exclusively) found in two clinical settings - primary infant acute leukemia and AML arising in patients previously treated with topoisomerase II inhibitors [53]. MLL encodes a protein that is part of a complex that regulates histone methylation (hence its new designation), and translocations involving $M L L$ lead to dysregulated histone modification and chromatin accessibility [54]. Unlike most loci involved in AML-associated translocations, in which both fusion partners are unique or show rare variants, $M L L$ has been found with more than 80 different partners [33]. The prognosis of patients with an $M L L$ translocation varies depending on the partnering locus. One large study evaluating outcomes in children with $M L L$-rearranged leukemia found poor prognostic associations with translocations involving chromosomes 6q27, 10p11.2, and 4q21 (overall survival 5-year survival of 22-27\%), while all 27 children with a $\mathrm{t}(1 ; 11)$ translocation were alive at 5 -year follow-up [55]. The most common translocation partner of $M L L$ in AML cases is MLLT3, located on chromosome 9. This $\mathrm{t}(9 ; 11)(\mathrm{p} 22 ; \mathrm{q} 23)$ abnormality occurs in about half of $M L L-$ rearranged AML cases and defines a WHO-described subcategory of AML. Some studies have suggested that children with this translocation carry a favorable prognosis, though others have shown intermediate outcomes [30, 32, 55, 56].

A few additional translocations are not included in the current WHO classification but are recurrently found in pediatric AML and merit discussion. Fusions involving NUP98 (such as the NUP98-KDM5A fusion previously discussed) are recurrently found in pediatric AML. Like MLL, NUP98 is nondiscriminatory and associates with multiple different partners 
[57]. One such fusion involves NUP98 and the nuclear receptor domain SET binding protein 1 gene (NSD1), which results from $\mathrm{t}(5 ; 11)(\mathrm{q} 35 ; \mathrm{p} 15.5)$ [58-60]. This translocation is cytogenetically cryptic and has been found in $16 \%$ of karyotypically normal childhood AML. Interestingly, patients with this translocation frequently harbor concomitant internal tandem duplications in the fms-related tyrosine kinase 3 (FLT3) gene and have a relatively poor prognosis. Likewise, AML with a $\mathrm{t}(8 ; 16)(\mathrm{p} 11 ; \mathrm{p} 13)$ translocation is also associated with a poor prognosis $[61,62]$. This translocation is seen infrequently in cases of infant AML and has the characteristic cytologic finding of blasts with hemophagocytosis. Despite the relatively aggressive nature of this AML, a subset of patients shows spontaneous regression and long-term remission of their leukemia, a phenomenon somewhat reminiscent of the transient abnormal myeloproliferation seen in a subset of infants with Down syndrome.

Numerical abnormalities involving gains or losses of chromosomal material are also particularly important in determining prognosis of patients with AML. Complex karyotypes (currently defined as three or more chromosomal abnormalities not otherwise associated with an AML subtype) as well as a monosomy karyotype (defined as monosomy of two chromosomes or a single monosomy in the context of other structural abnormalities) both portend a poor prognosis $[33,63$, 64]. In adults, loss of chromosome 7 or 7q is also associated with aggressive disease [65]. These last abnormalities occur infrequently in children and should not be seen as a single entity since children with $7 \mathrm{q}$ loss have a significantly better prognosis than those with loss of the entire chromosome 7 [66]. Moreover, additional chromosomal abnormalities when present with -7 , especially inv(3), -5 , and +21 , have a significant negative impact on patient outcome.

\section{Clinical assessment of "large-scale" changes in AML}

Many of the genomic changes in AML discussed above were initially recognized by standard metaphase chromosomal analysis [10]. Despite remarkable advances in profiling human genomes, this seemingly antiquated technique remains a cornerstone of diagnostic testing for patients with AML [67]. The technique is well-established and relatively straightforward yet requires highly skilled cytogeneticists to perform the assay and interpret the data. Most translocations in AML can be identified cytogenetically, though cryptic or noncanonical translocations requiring alternative methodologies for detection are not infrequent. Perhaps the greatest utility of cytogenetic analysis is that it gives a relatively unbiased view of the entire genome. Large-scale abnormalities may be detected, regardless if they were expected prior to performing the assay. This broad evaluation of tumor cell chromosomes is especially useful in diagnosing AML with complex karyotypes for which multiple abnormalities can be visualized with a single cellular preparation.

A second method for analyzing large-scale genomic alterations is fluorescence in situ hybridization or FISH [68]. With this technique, fluorescent DNA probes are hybridized to the loci of interest within the nuclei of sample material. The resulting fluorescent signals reflect the absolute number and the relative chromosomal location of the loci in question. Standard probes used clinically for translocation analysis can show juxtaposition to two loci (fusion probes), splitting of a single locus (break-apart probes), or both (dual-fusion probes). For diagnostic samples, in which there is a preponderance of tumor cells, FISH is the current gold standard for detecting most translocations since its sensitivity approaches $100 \%$ and can detect cryptic fusions potentially missed by standard cytogenetics or reverse transcription polymerase chain reaction (RT-PCR)-based assays [68, 69]. An additional useful characteristic of chromosomal analysis by FISH is that it does not require cells to be alive and cycling, whereas standard cytogenetics does. Thus, diagnostic samples that are unable to grow in the laboratory or that are formalin fixed can still be analyzed for recurrent genetic abnormalities by FISH [70]. Broad genomic analysis by FISH, however, is much more limited than by standard cytogenetics. A general overview of the chromosomal composition of AML cells is not possible with standard diagnostic FISH techniques since only a restricted set of FISH probes is used. FISH in isolation, therefore, may allow for detection of specific - and suspected-genetic events but can easily miss other unsuspected - and perhaps prognostically important - chromosomal abnormalities. Clearly, the sensitivity of FISH and the broad chromosomal analysis of standard cytogenetics complement one another, and both techniques are (and should be) parts of a standard workup of diagnostic samples from patients with AML.

Despite the diagnostic sensitivity of these two techniques (especially FISH) in identifying chromosomal lesions, their analytic sensitivity is quite low. In a sample being analyzed by FISH, usually only $200-500$ cells are examined microscopically for aberrant signals [68]. Standard karyotypes involve examination of only 20 cells. However, detection of minimal residual disease, which is clinically meaningful with blast percentages of 1 in 10,000 cells, obviously requires analysis of many more cells than can be reasonably done using either of these two techniques [71]. Thus, both FISH and cytogenetics are invaluable tools at initial diagnosis and during frank cytologic recurrence (when blast percentages are relatively high), but they have virtually no role for monitoring patients who are in cytologic remission.

A commonly used alternative method for detecting translocations involves RT-PCR with primer pairs specific for the two loci involved in the translocation $[72,73]$. With this method, the presence of the fused loci results in the production of a 
fusion-specific PCR amplicon. Detection of the PCR product can be accomplished by multiple methodologies including gel or capillary electrophoresis and real-time amplification, the latter of which also allows for quantification of a relative amount of fusion DNA and may be useful for following patients in cytologic remission. A number of methodologies use multiplex PCR to screen for many common translocations in a single reaction. Products can be distinguished from one another by the size differences of the resulting amplicons or by hybridization to product specific oligonucleotides covalently coupled to fluorescent microspheres [74, 75].

PCR techniques have a number of advantages over both FISH and standard cytogenetics to identify chromosomal translocations. PCR has the fastest turnaround time of these three techniques, so is the method of choice when a rapid diagnosis is essential (e.g., AML with PML-RARA). PCR also has the highest analytic sensitivity, so these methods can be used to detect leukemic cells during and after treatment, when evaluation of minimal residual disease is a central clinical question [76].

Although PCR-based techniques have certain advantages, they are not entirely without flaws. The exquisite analytic sensitivity of PCR, one of the main assets of the technique, is ironically one of its drawbacks as well. Low levels of AMLassociated fusions can be detected in DNA samples from patients without leukemia, and patients can remain in long-term remission while continuing to display PCR-positivity. Thus, a positive PCR result is neither specific for leukemia nor does it not indicate treatment failure [72, 77-80]. Along these same lines, the presence of residual blasts below 1 in 10,000 may not be clinically relevant, so the ability of PCR to detect cells as low as one in a million may be excessive. Additionally, the use of PCR with translocation-specific probes to follow patients during treatment is restricted to patients who have a detectable abnormality at diagnosis; these represent only a subset of AML patients. Given these drawbacks, multiparameter flow cytometry, which can detect immunophenotypic abnormalities in most cases of AML and can do so with an appropriate analytic sensitivity of $0.01 \%$, is typically used to follow pediatric patients with AML during their treatment [71].

\section{Submicroscopic (small-scale) mutations in AML}

Initial identification of genomic abnormalities in AML was based on rather blunt techniques for assessing the human genome. As techniques for the rapid assessment of nucleotide sequence have become widespread, numerous additional mutations have been found to be repeatedly associated with AML [6]. Like the large-scale genomic alterations, many of these small-scale mutations, which include point mutations and small insertions and deletions, have prognostic implications, and a few have been incorporated into formal classification schemata.

Of these small-scale mutations, two can be used for defining subclasses of AML (albeit provisional) using the most recent WHO classification guidelines. These are AML with mutations in nucleophosmin (NPMI) and AML with mutations in the gene encoding the CCAAT/enhancer-binding protein alpha (CEBPA) [6].

Recurrent mutations in NPM1 in AML were first recognized by Falini and colleagues [81]. NPM1, which is largely found in the nucleolus, is thought to have a role in ribosomal biogenesis and may also have a link to tumor suppression by aiding in nucleolar sequestration of the MDM2 oncoprotein [82-84]. Mutations in NPM1, which are typically found in the C-terminus, abrogate the nucleolar localization signal, and the protein is allowed to migrate to the cytoplasm. Indeed, a rapid test for determining if an NPM1 mutation exists is a simple immunohistochemical stain against NPM1 protein and determining microscopically if the protein localizes outside of the nucleus [85].

NPM1 mutations are found in $~ 30 \%$ of adult AML, yet only about $10 \%$ of pediatric AML cases possess NPM1 mutations [86-88]. Mutations in NPM1 are most commonly found in AML with a normal karyotype, and a significant overlap can be found between NPM1 mutations and activating mutations in the FLT3 gene [89]. This relationship between NPM1 and FLT3 mutations is important with regard to patient prognosis. AML with an NPM1 mutation portends a rather favorable prognosis, but only when a concomitant activating internal tandem duplication in FLT3 is not detected (see below) $[15,87,90,91]$. Further studies have indicated that NPM1 mutations are associated with favorable prognosis only when they occur simultaneously with isocitrate dehydrogenase $(I D H)$ mutations [15], yet these results remain to be verified in independent AML cohorts.

The second entity with provisional diagnostic status in the most recent WHO classification is AML with CEBPA mutations [6]. This gene encodes a transcription factor that is critical for myeloid differentiation [92], and consistent with the lack of maturation seen in AML blasts, mutation abrogates $C E B P A$ function [93]. In adults, tumors with CEBPA mutations also have a relatively good prognosis, but this seems only true for cases with biallelic mutations [93-95]. These prognostic features have been difficult to validate in the pediatric population, as the mutation is present in only $\sim 5 \%$ of childhood AML, but recent studies are consistent with survival benefit of a biallelic CEBPA mutation [96].

Many of the growth-promoting mutations in AML are activating point mutations in receptor tyrosine kinases or downstream molecules. These include activating mutations in $K I T$, $N R A S$, and KRAS. Of particular interest in AML, particularly due to the relatively high mutational frequency, impact on prognosis, and recent development of small-molecule 
inhibitors, are activating mutations in the FLT3 gene. Mutations in FLT3 are found in $25 \%$ of AML and are common in AML with normal karyotype [97, 98]. AML-associated FLT3 mutations result in constitutive kinase activity, but occur in two different flavors. The majority of mutations are internal tandem duplications (termed FLT3-ITD) in the juxtamembrane domain, while a minority are point mutations in the kinase domain (FLT3-KD) [90, 97, 99]. These mutations are somewhat puzzling with regard to patient outcome and disease biology. FLT3-ITD is clearly associated with poor patient outcomes [97, 98, 100-102]; surprisingly, the negative prognostic implications do not hold for FLT3-KD mutations as a number of studies have shown that FLT3-KD-mutant tumors are not as aggressive as their FLT3-ITD counterparts $[103,104]$. These results suggest that constitutive kinase activity may not be the only disruption caused by the internal duplication.

FLT3 mutations are thought to be acquired late during tumorigenesis; therefore, the mutational burden of FLT3-ITD can vary widely since only a subset of the original clones possesses the mutation. In fact, prognosis of FLT3-ITD tumors in both the adult and pediatric population has been shown to correlate with allele burden [90, 97]. Additionally, discrepancies in FLT3-status between diagnostic and relapse samples may exist, in that some patients with FLT3-ITD diagnostic samples will lack the mutation at relapse and vice versa [105]. Given that FLT3-ITD is typically associated with an aggressive phenotype, gain of the mutation at relapse may be expected but its loss at relapse is rather surprising.

Interestingly, studies in which cases of AML were segregated based on either transcriptional or DNA methylation profiling identified subgroups with significant enrichment of CEBPA mutation and NPM1 mutations [106, 107]. Additional subgroups enriched for chromosomal abnormalities [such as $\mathrm{t}(15 ; 17)$ and $\mathrm{t}(8 ; 21)]$ were also identified. By contrast, FLT3 mutations were not confined to certain subgroups but were found across the sample sets. These studies are consistent with the hypothesis that FLT3 mutations are acquired late in tumorigenesis and that the recurrent translocations and mutations of CEBPA and NPM1 are disease-initiating events, while FLT3 mutations modify tumor aggressiveness (Tables 1 and 2).

Given the prognostic discrepancies between FLT3-ITD and FLT3-KD mutations, the cases of tumor recurrence with loss of FLT3-ITD, and the relatively late acquisition of FLT3 mutations in tumorigenesis, it is tempting to hypothesize that a FLT3 mutation may not be a driver mutation in AML, but may actually represent a marker aggressive disease. Arguing against this model, Smith et al., in a number of elegant experiments, showed that patients with FLT3-ITD blasts and treated with FLT3 inhibitors acquired additional mutations in FLT3 that rendered the kinase insensitive to inhibition [117]. These results suggest that FLT3-ITD (and its inhibition) exerts a selective pressure on AML blasts and is, therefore, partially driving the disease rather than merely acting as a disease marker. The fact that FLT3-ITD is likely a driver mutation in AML, coupled with the high mutation frequency and its effect on patient prognosis, has made it a key target for development and testing of AML therapeutics in both the adult and pediatric populations [4, 118].

Since the publication of the WHO classification in 2008, many additional recurrent mutations in AML have been discovered. This wave of novel AML mutations has been driven in large part by the advent of next-generation sequencing technology, which allows relatively rapid exome or genome-wide screening of many AML samples. Of the recently discovered AML-associated mutations, much attention has focused on loci that are involved in the regulation of epigenetic events, such as DNA (cytosine-5-)-methyltransferase 3 alpha $(D N M T 3 A)$, tet methylcytosine dioxygenase 2 (TET2) and IDH1, and IDH2 [14-16, 18, 108, 115]. All of these mutations cause dysregulation of DNA methylation. DNMT3A is a maintenance DNA methyltransferase. Mutations in this gene, found in 20\% of adult AML, dominantly abrogate methyltransferase function [17, 119]. TET2 is involved in DNA hydroxymethylation, an intermediate step in demethylation of DNA, and mutations in TET2 also alter DNA methylation profiles [109]. IDH1 and IDH2 mutations are rather interesting since they create a neo-enzymatic activity that increases the cellular production of 2-hydroxyglutarate (2HG) $[120,121]$. 2-HG inhibits the function of TET2, similarly altering the methylation profiles of AML. As would be expected with mutations in the same functional pathway, the subset of AML cases with TET2 mutations is almost entirely nonoverlapping with the subset of tumors with IDH1 or IDH2 mutations [109]. Prognostically, patients with TET2 mutations seem to have more aggressive disease compared to those patients without these mutations [15, $113,114]$. The data are less clear for DNMT3A, IDH1, and IDH2 mutations [108].

Next-generation sequencing not only has allowed for the identification and study of mutations at specific loci but also has allowed the entire mutational profile of multiple AML cases to be cataloged. In fact, TCGA studies lead by the sequencing group at Washington University have recently completed the mutational status of 200 adult AML diagnostic samples [14, 122]. These studies have revealed interesting insights into both the number and the types of AML mutations, particularly when compared to other tumor classes. AML samples, on average, have significantly fewer mutations than most other adult tumor types including lung, colon, breast, and melanoma [122]. In fact, the total number of mutations in adult AML is more similar to those of pediatric tumors such as neuroblastoma. These large-scale sequencing studies have not only revealed common mutations in AML (such as DNMT3A) but have also highlighted what mutations 
Table 1 Recurrent translocations found in AML discussed in the text

\begin{tabular}{|c|c|c|c|c|}
\hline Genetic abnormality & $\begin{array}{l}\text { Frequency } \\
\text { in adult } \\
\text { AML }\end{array}$ & $\begin{array}{l}\text { Frequency } \\
\text { in pediatric } \\
\text { AML }\end{array}$ & Comments & References \\
\hline $\begin{array}{l}P M L-R A R A \\
\quad \mathrm{t}(15 ; 17)(\mathrm{q} 24 ; \mathrm{q} 21)\end{array}$ & $\sim 10 \%$ & $\sim 10 \%$ & $\begin{array}{l}\text { Strong correlation with } \\
\text { FAB M3 (APL) } \\
\text { Rare variant translocations } \\
\text { Rapid diagnosis essential } \\
\text { Treatment with ATRA } \\
\text { Favorable prognosis }\end{array}$ & {$[10,20,24-30,32]$} \\
\hline $\begin{array}{l}R U N X 1-R U X N 1 T 1 \\
\quad \mathrm{t}(8 ; 21)(\mathrm{q} 22 ; \mathrm{q} 22.3)\end{array}$ & $5-10 \%$ & $10-15 \%$ & $\begin{array}{l}\text { Correlation with FAB M2 } \\
\text { Correlation with } \\
\text { granulocytic sarcomas } \\
\text { Favorable prognosis }\end{array}$ & $\begin{array}{l}{[10,20,30} \\
\quad 32,35,36]\end{array}$ \\
\hline $\begin{array}{l}\text { CBFB-MYH11 } \\
\quad \operatorname{inv}(16)(\mathrm{p} 13.1 \mathrm{q} 22) \\
\text { or } \\
\text { Favorable prognosis }\end{array}$ & $5-10 \%$ & $\begin{array}{l}\text { Correlation with } \\
\text { FAB M4eo }\end{array}$ & $\begin{array}{c}\mathrm{t}(16 ; 16)(\mathrm{p} 13.1 ; \mathrm{q} 22) \\
{[10,20,30,32,37,38]}\end{array}$ & $\sim 5 \%$ \\
\hline $\begin{array}{l}K M T 2 A \\
\text { previously } M L L \\
\quad 11 \mathrm{q} 23\end{array}$ & $\sim 5 \%$ & $15-20 \%$ & $\begin{array}{l}\text { Most frequent in infants } \\
\text { Many translocation partners } \\
\text { Prognosis varies with } \\
\text { translocation partner } \\
\text { Usually poor prognosis }\end{array}$ & $\begin{array}{l}{[10,20,30,32,} \\
\quad 55,56]\end{array}$ \\
\hline $\begin{array}{l}D E K-N U P 214 \\
\mathrm{t}(6 ; 9)(\mathrm{p} 23 ; \mathrm{q} 34)\end{array}$ & $\sim 1 \%$ & $\sim 1 \%$ & $\begin{array}{l}\text { Frequent myelodysplasia } \\
\text { and basophilia } \\
\text { Poor prognosis }\end{array}$ & {$[41,42]$} \\
\hline $\begin{array}{l}R P N 1-M E C O M \\
\operatorname{inv}(3)(\mathrm{q} 21 \mathrm{q} 26.2) \\
\text { or } \mathrm{t}(3 ; 3)(\mathrm{q} 21 ; \mathrm{q} 26.2)\end{array}$ & $1-2 \%$ & Rare & $\begin{array}{l}\text { Frequent myelodysplasia } \\
\text { and atypical } \\
\text { megakaryocytes } \\
\text { Poor prognosis }\end{array}$ & [46-49] \\
\hline $\begin{array}{l}R B M 15-M K L 1 \\
\mathrm{t}(1 ; 22)(\mathrm{p} 13 ; \mathrm{q} 13)\end{array}$ & Rare & $\begin{array}{l}\sim 15 \% \text { of } \\
\text { AMKL } \\
\text { cases }\end{array}$ & $\begin{array}{l}\text { Mostly infants with } \\
\text { AMKL } \\
\text { Relatively favorable } \\
\text { prognosis }\end{array}$ & {$[6,30,32,50]$} \\
\hline $\begin{array}{l}\text { NUP98-KDM5A } \\
\mathrm{t}(11 ; 12)(\mathrm{p} 15 ; \mathrm{p} 13)\end{array}$ & NA & $\begin{array}{l}\sim 10 \% \text { of } \\
\text { AMKL } \\
\text { cases }[50]\end{array}$ & $\begin{array}{l}\text { Limited studies } \\
\text { No clear association } \\
\text { with prognosis }\end{array}$ & {$[50]$} \\
\hline $\begin{array}{l}\text { CBFA2T3-GLIS2 } \\
\operatorname{inv}(16)(\mathrm{p} 13.3 \mathrm{q} 24.3)\end{array}$ & NA & $\begin{array}{r}15-25 \% \\
\text { AMKL }\end{array}$ & $\begin{array}{l}\text { Limited studies } \\
\text { Initial report showed } \\
\text { poor prognosis }\end{array}$ & {$[50,51]$} \\
\hline $\begin{array}{l}\text { NUP98-NSD1 } \\
\mathrm{t}(5 ; 11)(\mathrm{q} 35 ; \mathrm{p} 15.5)\end{array}$ & $1-2 \%$ & $4-7 \%$ & $\begin{array}{l}\text { Cytogenetically cryptic } \\
\text { Poor prognosis }\end{array}$ & {$[58-60]$} \\
\hline $\begin{array}{l}\text { MYST3-CREBBP } \\
\mathrm{t}(8 ; 16)(\mathrm{p} 11 ; \mathrm{p} 13)\end{array}$ & NA & $<1 \%$ & $\begin{array}{l}\text { Mostly in infants } \\
\text { Associated with } \\
\text { erythrophagocytosis } \\
\text { Few cases show } \\
\text { spontaneous remission } \\
\text { Poor prognosis }\end{array}$ & {$[61,62]$} \\
\hline $\begin{array}{l}\text { Loss of chromosome } \\
7\end{array}$ & $\sim 5 \%$ & $2-5 \%$ & Poor prognosis & [64-66] \\
\hline Complex karyotype & $10-15 \%$ & $5-10 \%$ & Poor prognosis & {$[10,20,32]$} \\
\hline
\end{tabular}

are noticeably underrepresented. Strikingly, the mutational spectrum of AML - dominated by the loci discussed above - is remarkably distinct from the mutational spectrum of solid tumors, which is significantly weighted toward the well-described tumor suppressors TP53,
$C D K N 2 A$, and ATM. These studies indicate that the selective pressures involved in forming AML are distinct from those involved in generating a solid tumor, and they should provide insight for designing therapeutics to target genes involved in tumor selection. 
Table 2 Recurrent mutations found in AML discussed in the text

\begin{tabular}{|c|c|c|c|c|}
\hline Mutated gene & $\begin{array}{l}\text { Frequency in } \\
\text { adult AML (\%) }\end{array}$ & $\begin{array}{l}\text { Frequency in } \\
\text { pediatric AML }(\%)\end{array}$ & Comments & References \\
\hline NPM1 & $\sim 30$ & $\sim 10$ & $\begin{array}{l}\text { Mutation results in cytoplasmic } \\
\text { localization of protein } \\
\text { Common in AML with normal karyotype } \\
\text { Associated with FLT3-ITD } \\
\text { Favorable prognosis if FLT3 is wild-type }\end{array}$ & {$[85-91]$} \\
\hline FLT3 & $\sim 25$ & $\sim 15$ & $\begin{array}{l}\text { Two types of mutations: ITD and KD } \\
\text { FLT3-ITD associated with poor outcomes } \\
\text { Allele burden influences prognosis } \\
\text { Many cases with discrepant FLT3-ITD } \\
\text { status a diagnosis and relapse }\end{array}$ & [97-102] \\
\hline CEBPA & $5-10$ & $\sim 5$ & Favorable prognosis if both alleles mutated & {$[92-96]$} \\
\hline$I D H 1 / 2$ & $\sim 20$ & $<5$ & $\begin{array}{l}\text { Mutations create neo-enzymatic activity } \\
\text { Inhibits hydroxymethylation of DNA } \\
\text { Alteration of DNA methylation profile of AML } \\
\text { Conflicting data on correlation with outcome } \\
\text { Mostly mutually exclusive with TET2 mutations }\end{array}$ & {$[14,108-112]$} \\
\hline DNMT3A & $\sim 20$ & $<5$ & $\begin{array}{l}\text { Mutations create dominant negative form of DNMT3A } \\
\text { Alteration of DNA methylation profiles }\end{array}$ & {$[14,18,110,111,113,114]$} \\
\hline TET2 & $5-10$ & $<5$ & $\begin{array}{l}\text { Influences hydroxymethylation and demethylation of DNA } \\
\text { Similar DNA methylation profiles as } \\
\text { IDH1/2 mutant AML } \\
\text { Possibly associated with poor prognosis }\end{array}$ & {$[14,110,111,115,116]$} \\
\hline
\end{tabular}

Clinical assessment of "small-scale" changes in AML

Detection of point mutations or small insertions and deletions in specific loci relies heavily on PCR amplification. The choice of PCR primers and the method of analysis of PCR amplicons influence both the diagnostic and the analytic sensitivity of the chosen assay. PCR primers can be designed to be specific for the mutant allele (i.e., allele-specific oligonucleotides or ASOs) so that a product is formed only when the mutant exists [123]. This assay method is virtually identical to PCR analysis of specific translocations and is subject to the same advantages and disadvantages. The analytic sensitivity is quite high, especially if real-time detection methods are used for analysis, yet its diagnostic sensitivity is not $100 \%$ and care should be used when interpreting positive results as they may not entirely reflect clinical status.

PCR with primers that flank regions of interest followed by amplicon detection by methods that can distinguish between wild-type and mutant alleles is another method of determining the mutational status of AML-associated loci [124, 125]. This method is typically used for FLT3-ITD evaluation, in which primers flanking the ITD are used in the PCR, and electrophoresis is used to show an increase in amplicon size when the ITD is present. These methods have a higher diagnostic sensitivity than ASO-based methods, as they are not restricted by a specific mutation. However, since they produce an amplicon regardless of mutational status, the wild-type allele can dominate the analysis in samples with low mutational burden. Thus, the analytic sensitivity of the assay is lower than that of ASO-PCR.

The above PCR methods are typically used with a single or relatively few loci. Given the growing array of mutations involved in AML, many institutions have recently begun to explore the utility of using massively parallel, nextgeneration sequencing (NGS) to evaluate the entire mutational profile of AML cells [126]. Since most of the mutations in AML are known, and only a subset of these are actionable or have prognostic relevance, sequencing panels are used by many institutions. Limiting the number of loci evaluated, rather than broadly evaluating the entire exome or genome, increases both the speed and analytic sensitivity of mutational assessment and limits the costs involved

Most of the information that can be gleaned from multiple other molecular techniques can be obtained from simply using a relatively restricted panel of loci in a NGS assay. Clearly, point mutations, small insertions, and deletions can be detected by this sequencing methodology. Using read counts of each locus, the allele frequency and locus copy number (which may indicate chromosomal gains and losses) can be determined. Similarly, since allele frequency is determined digitally through read counts and is not subject to the normal allele dilutional effects of analog methods, NGS with high read- 
depth can be used for determination of minimal residual disease. Sequences that span translocation breakpoints can be used to determine the presence of translocations and inversions. Given these characteristics, NGS assays are likely to supplant traditional single-locus assays for mutational analysis of AML.

Mutational analysis and development of tests that probe DNA mutations has clearly dominated the field of molecular analysis of AML. Mutations, however, likely do not account for all of the dysregulation involved in tumorigenesis and probing other, non-mutational events may have significance in guiding treatment or determining the prognosis of patients with AML. As briefly mentioned above, dysregulation of both the transcriptional and epigenetic profiles is characteristic of AML and multilocus assessment of gene transcription, or DNA methylation has been shown to segregate tumors into specific subgroups with unique genetic and clinical features [106, 107, 127, 128]. Indeed, studies of DNA methylation in adult AML have shown that methylation patterns have prognostic significance independent of currently used clinical and genetic parameters. Although methods for assessing transcriptional profiles and DNA methylation status are not currently used in clinical evaluation of patients with AML, they are used for patients with other types of tumors [129, 130], and a number of groups are actively pursuing methods for nonmutational molecular tests in leukemic samples [131, 132].

The research on the molecular events involved in AML leukemogenesis has far outpaced the translation of important findings into clinical decision-making. Current recommendations of genetic testing in pediatric AML include karyotypic analysis, targeted assessment of critical translocations [i.e., $\mathrm{t}(15 ; 17), \mathrm{t}(8 ; 21), \operatorname{inv}(16) / \mathrm{t}(16 ; 16)$, and $11 \mathrm{q} 23]$ as well as NPM1, FLT3, and CEBPA mutational status [133]. Further testing based on specific clinical or cytologic features (such as hemophagocytic blasts or megakaryocytic differentiation) should also be pursued on a case-by-case basis.

\section{Pediatric AML vs. adult AML, are they identical?}

Studying the effects of age on the biology and the clinical aspects of tumors is generally restricted to relatively small age ranges since most tumors occur either in the pediatric setting (e.g., neuroblastoma) or in the adult setting (e.g., non-small cell lung carcinoma) alone. The incidence of AML across all ages, however, provides a unique opportunity to study the effect of age on tumorigenesis and the mutational events that are prevalent in specific age-defined populations. Cytologically, adult and pediatric AML have similar features and, for the most part, do not vastly differ in their prevalence of cytogenetic alterations [134].

However, the distinction between adult and pediatric AML with regard to mutations in genes that influence the epigenome is quite striking. As noted earlier, the MLL gene, which is involved in histone modifications, shows a high prevalence of mutation in infant AML. Mutations in DNMT3A occur in approximately $20 \%$ of adult AML but are rarely found in pediatric AML $[110,111,134]$. Similarly, IDH1 and $I D H 2$ mutations are found in $10-15 \%$ of adult AML but are much rarer in pediatric AML. Since mutations in epigenetic modifiers are likely early events in leukemogenesis, one can hypothesize that alteration of the epigenetic landscape is an initial requirement for leukemogenesis. The discrepant frequencies of these mutations between adult and pediatric AML may indicate that these are fundamentally distinct sets of tumors when viewed epigenetically. Alternatively, one could hypothesize that the epigenetic landscape of the physiologic hematopoietic stem cell is different between adults and children, and that different mutations are initially required to establish a common leukemic epigenetic profile. Establishing the underlying reasons for the striking differences in the mutational profiles of adult and pediatric AML would certainly add insight into leukemogenic pathways and could guide the modulation of treatment paradigms depending on patient age.

Acknowledgments GBWW is supported, in part, by a pilot project grant from the Perelman School of Medicine Hematologic Malignancies Translational Center of Excellence, the UPenn Institute for Translational Medicine and Therapeutics (ITMAT) through a grant from the National Center for Research Resources, Grant UL1RR024134 (now at the National Center for Advancing Translational Sciences, Grant UL1TR000003), and by grant IRG-78-002-35 from the American Cancer Society.

Conflict of interest The author declares no conflict of interest.

\section{References}

1. Deschler B, Lubbert M (2006) Acute myeloid leukemia: epidemiology and etiology. Cancer 107(9):2099-2107

2. Meshinchi S, Arceci RJ (2007) Prognostic factors and risk-based therapy in pediatric acute myeloid leukemia. Oncologist 12(3): 341-355

3. Pui CH et al (2011) Biology, risk stratification, and therapy of pediatric acute leukemias: an update. J Clin Oncol 29(5):551-565

4. Tasian SK, Pollard JA, Aplenc R (2014) Molecular therapeutic approaches for pediatric acute myeloid leukemia. Front Oncol 4: 55

5. Bitter MA et al (1987) Associations between morphology, karyotype, and clinical features in myeloid leukemias. Hum Pathol 18(3):211-225

6. Swerdlow SH, Campo E, Harris NL, Jaffe ES, Pileri SA, Stein H, Thiele J, Vardiman J (2008) WHO classification of tumours of haematopoietic and lymphoid tissue. International Agency for Research on Cancer, Lyon

7. Bennett JM et al (1976) Proposals for the classification of the acute leukaemias. French-American-British (FAB) co-operative group. Br J Haematol 33(4):451-458

8. Burke MJ et al (2014) Similar outcomes between adolescent/ young adults and children with AML following allogeneic hematopoietic cell transplantation. Bone Marrow Transplant 49(2): $174-178$ 
9. Canner J et al (2013) Differences in outcomes of newly diagnosed acute myeloid leukemia for adolescent/young adult and younger patients: a report from the Children's Oncology Group. Cancer 119(23):4162-4169

10. Grimwade D et al (1998) The importance of diagnostic cytogenetics on outcome in AML: analysis of 1,612 patients entered into the MRC AML 10 trial. The medical research council adult and children's leukaemia working parties. Blood 92(7):2322-2333

11. Deguchi K, Gilliland DG (2002) Cooperativity between mutations in tyrosine kinases and in hematopoietic transcription factors in AML. Leukemia 16(4):740-744

12. Kelly LM, Gilliland DG (2002) Genetics of myeloid leukemias. Annu Rev Genomics Hum Genet 3:179-198

13. Dombret H (2011) Gene mutation and AML pathogenesis. Blood 118(20):5366-5367

14. The Cancer Genome Atlas Research Network (2013) Genomic and epigenomic landscapes of adult de novo acute myeloid leukemia. N Engl J Med, 368(22): 2059-2074

15. Patel JP et al (2012) Prognostic relevance of integrated genetic profiling in acute myeloid leukemia. N Engl J Med 366(12): 1079-1089

16. Rocquain $\mathrm{J}$ et al (2010) Combined mutations of ASXL1, CBL, FLT3, IDH1, IDH2, JAK2, KRAS, NPM1, NRAS, RUNX1, TET2 and WT1 genes in myelodysplastic syndromes and acute myeloid leukemias. BMC Cancer 10:401

17. Wertheim GB, Hexner E, Bagg A (2012) Molecular-based classification of acute myeloid leukemia and its role in directing rational therapy: personalized medicine for profoundly promiscuous proliferations. Mol Diagn Ther 16(6):357-369

18. Ley TJ et al (2010) DNMT3A mutations in acute myeloid leukemia. N Engl J Med 363(25):2424-2433

19. Mardis ER et al (2009) Recurring mutations found by sequencing an acute myeloid leukemia genome. N Engl J Med 361(11):10581066

20. Tallman MS (1994) All-trans-retinoic acid in acute promyelocytic leukemia and its potential in other hematologic malignancies. Semin Hematol 31(4 Suppl 5):38-48

21. Labrecque $J$ et al (1998) Impaired granulocytic differentiation in vitro in hematopoietic cells lacking retinoic acid receptors alpha1 and gamma. Blood 92(2):607-615

22. Purton LE et al (2006) RARgamma is critical for maintaining a balance between hematopoietic stem cell self-renewal and differentiation. J Exp Med 203(5):1283-1293

23. Sanz MA et al (2010) Risk-adapted treatment of acute promyelocytic leukemia based on all-trans retinoic acid and anthracycline with addition of cytarabine in consolidation therapy for high-risk patients: further improvements in treatment outcome. Blood 115(25):5137-5146

24. Grimwade D et al (2010) Refinement of cytogenetic classification in acute myeloid leukemia: determination of prognostic significance of rare recurring chromosomal abnormalities among 5876 younger adult patients treated in the United Kingdom Medical Research Council trials. Blood 116(3):354-365

25. Redner RL et al (1996) The $\mathrm{t}(5 ; 17)$ variant of acute promyelocytic leukemia expresses a nucleophosmin-retinoic acid receptor fusion. Blood 87(3):882-886

26. Wells RA, Catzavelos C, Kamel-Reid S (1997) Fusion of retinoic acid receptor alpha to NuMA, the nuclear mitotic apparatus protein, by a variant translocation in acute promyelocytic leukaemia. Nat Genet 17(1):109-113

27. Rohr SS et al (2012) Acute promyelocytic leukemia associated with the PLZF-RARA fusion gene: two additional cases with clinical and laboratorial peculiar presentations. Med Oncol 29(4): 2345-2347
28. Sainty D et al (2000) A new morphologic classification system for acute promyelocytic leukemia distinguishes cases with underlying PLZF/RARA gene rearrangements. Blood 96(4):1287-1296

29. Arnould $C$ et al (1999) The signal transducer and activator of transcription STAT5b gene is a new partner of retinoic acid receptor alpha in acute promyelocytic-like leukaemia. Hum Mol Genet 8(9):1741-1749

30. Harrison CJ et al (2010) Cytogenetics of childhood acute myeloid leukemia: United Kingdom Medical Research Council Treatment trials AML 10 and 12. J Clin Oncol 28(16):2674-2681

31. Rubnitz JE et al (2010) Minimal residual disease-directed therapy for childhood acute myeloid leukaemia: results of the AML02 multicentre trial. Lancet Oncol 11(6):543-552

32. von Neuhoff $\mathrm{C}$ et al (2010) Prognostic impact of specific chromosomal aberrations in a large group of pediatric patients with acute myeloid leukemia treated uniformly according to trial AML-BFM 98. J Clin Oncol 28(16):2682-2689

33. Morrissette JJ, Bagg A (2011) Acute myeloid leukemia: conventional cytogenetics, FISH, and moleculocentric methodologies. Clin Lab Med 31(4):659-686, x

34. Walter $\mathrm{K}$ et al (2010) Aberrant expression of CD19 in AML with $\mathrm{t}(8 ; 21)$ involves a poised chromatin structure and PAX5. Oncogene 29(20):2927-2937

35. Schwyzer R et al (1998) Granulocytic sarcoma in children with acute myeloblastic leukemia and $t(8 ; 21)$. Med Pediatr Oncol 31(3):144-149

36. Tallman MS et al (1993) Granulocytic sarcoma is associated with the 8;21 translocation in acute myeloid leukemia. J Clin Oncol 11(4):690-697

37. Larson RA et al (1986) Acute myelomonocytic leukemia with abnormal eosinophils and inv(16) or $\mathrm{t}(16 ; 16)$ has a favorable prognosis. Blood 68(6):1242-1249

38. Le Beau MM et al (1983) Association of an inversion of chromosome 16 with abnormal marrow eosinophils in acute myelomonocytic leukemia. A unique cytogeneticclinicopathological association. N Engl J Med 309(11):630-636

39. Paschka $P$ et al (2006) Adverse prognostic significance of KIT mutations in adult acute myeloid leukemia with inv(16) and $\mathrm{t}(8 ; 21)$ : a Cancer and Leukemia Group B Study. J Clin Oncol 24(24):3904-3911

40. Pollard JA et al (2010) Prevalence and prognostic significance of KIT mutations in pediatric patients with core binding factor AML enrolled on serial pediatric cooperative trials for de novo AML. Blood 115(12):2372-2379

41. Tarlock K et al (2014) Acute myeloid leukaemia (AML) with $\mathrm{t}(6 ; 9)(\mathrm{p} 23 ; \mathrm{q} 34)$ is associated with poor outcome in childhood AML regardless of FLT3-ITD status: a report from the Children's Oncology Group. Br J Haematol 166(2):254-259

42. Alsabeh $\mathrm{R}$ et al (1997) Acute myeloid leukemia with $\mathrm{t}(6 ; 9)$ (p23;q34): association with myelodysplasia, basophilia, and initial CD34 negative immunophenotype. Am J Clin Pathol 107(4):430 437

43. Hoyle CF, Sherrington P, Hayhoe FG (1988) Translocation $(3 ; 6)(\mathrm{q} 21 ; \mathrm{p} 21)$ in acute myeloid leukemia with abnormal thrombopoiesis and basophilia. Cancer Genet Cytogenet 30(2): 261-267

44. Groschel S et al (2014) A single oncogenic enhancer rearrangement causes concomitant EVI1 and GATA2 deregulation in leukemia. Cell 157(2):369-381

45. Yamazaki H et al (2014) A remote GATA2 hematopoietic enhancer drives leukemogenesis in inv(3)(q21;q26) by activating EVI1 expression. Cancer Cell 25(4):415-427

46. Balgobind BV et al (2010) EVI1 overexpression in distinct subtypes of pediatric acute myeloid leukemia. Leukemia 24(5):942949 
47. Groschel S et al (2010) High EVI1 expression predicts outcome in younger adult patients with acute myeloid leukemia and is associated with distinct cytogenetic abnormalities. J Clin Oncol 28(12): 2101-2107

48. Groschel S et al (2013) Deregulated expression of EVI1 defines a poor prognostic subset of MLL-rearranged acute myeloid leukemias: a study of the German-Austrian Acute Myeloid Leukemia Study Group and the Dutch-Belgian-Swiss HOVON/SAKK Cooperative Group. J Clin Oncol 31(1):95-103

49. Lugthart $\mathrm{S}$ et al (2010) Clinical, molecular, and prognostic significance of WHO type inv(3)(q21q26.2)/t(3;3)(q21;q26.2) and various other $3 \mathrm{q}$ abnormalities in acute myeloid leukemia. J Clin Oncol 28(24):3890-3898

50. de Rooij JD et al (2013) NUP98/JARID1A is a novel recurrent abnormality in pediatric acute megakaryoblastic leukemia with a distinct HOX gene expression pattern. Leukemia 27(12):2280 2288

51. Gruber TA et al (2012) An Inv(16)(p13.3q24.3)-encoded CBFA2T3-GLIS2 fusion protein defines an aggressive subtype of pediatric acute megakaryoblastic leukemia. Cancer Cell 22(5):683-697

52. Masetti R et al (2013) CBFA2T3-GLIS2 fusion transcript is a novel common feature in pediatric, cytogenetically normal AML, not restricted to FAB M7 subtype. Blood 121(17):34693472

53. Tamai H, Inokuchi K (2010) 11q23/MLL acute leukemia : update of clinical aspects. J Clin Exp Hematop 50(2):91-98

54. Krivtsov AV, Armstrong SA (2007) MLL translocations, histone modifications and leukaemia stem-cell development. Nat Rev Cancer 7(11):823-833

55. Balgobind BV et al (2009) Novel prognostic subgroups in childhood 11q23/MLL-rearranged acute myeloid leukemia: results of an international retrospective study. Blood 114(12):2489-2496

56. Rubnitz JE et al (2002) Favorable impact of the $\mathrm{t}(9 ; 11)$ in childhood acute myeloid leukemia. J Clin Oncol 20(9):2302-2309

57. Braoudaki M, Tzortzatou-Stathopoulou F (2012) Clinical cytogenetics in pediatric acute leukemia: an update. Clin Lymphoma Myeloma Leuk 12(4):230-237

58. Akiki S et al (2013) NUP98-NSD1 fusion in association with FLT3-ITD mutation identifies a prognostically relevant subgroup of pediatric acute myeloid leukemia patients suitable for monitoring by real time quantitative PCR. Genes Chromosom Cancer 52(11):1053-1064

59. Hollink IH et al (2011) NUP98/NSD1 characterizes a novel poor prognostic group in acute myeloid leukemia with a distinct HOX gene expression pattern. Blood 118(13):3645-3656

60. Jaju RJ et al (2001) A novel gene, NSD1, is fused to NUP98 in the $\mathrm{t}(5 ; 11)(\mathrm{q} 35 ; \mathrm{p} 15.5)$ in de novo childhood acute myeloid leukemia. Blood 98(4):1264-1267

61. Coenen EA et al (2013) Pediatric acute myeloid leukemia with $\mathrm{t}(8 ; 16)(\mathrm{p} 11 ; \mathrm{p} 13)$, a distinct clinical and biological entity: a collaborative study by the International-Berlin-Frankfurt-Munster AML-study group. Blood 122(15):2704-2713

62. Hui CH, Suttle J (2001) Erythrophagocytosis associated with AML-M5 and $\mathrm{t}(8 ; 16)$. Br J Haematol 113(4):845

63. Breems DA et al (2008) Monosomal karyotype in acute myeloid leukemia: a better indicator of poor prognosis than a complex karyotype. J Clin Oncol 26(29):4791-4797

64. Kayser S et al (2012) Monosomal karyotype in adult acute myeloid leukemia: prognostic impact and outcome after different treatment strategies. Blood 119(2):551-558

65. van den Heuvel-Eibrink MM et al (2001) MDR1 expression in poor-risk acute myeloid leukemia with partial or complete monosomy 7. Leukemia 15(3):398-405
66. Hasle $\mathrm{H}$ et al (2007) Monosomy 7 and deletion $7 \mathrm{q}$ in children and adolescents with acute myeloid leukemia: an international retrospective study. Blood 109(11):4641-4647

67. Watt CD, Bagg A (2010) Molecular diagnosis of acute myeloid leukemia. Expert Rev Mol Diagn 10(8):993-1012

68. Wolff DJ et al (2007) Guidance for fluorescence in situ hybridization testing in hematologic disorders. J Mol Diagn 9(2):134-143

69. Frohling S et al (2005) Diagnostic value of fluorescence in situ hybridization for the detection of genomic aberrations in older patients with acute myeloid leukemia. Haematologica 90(2): 194-199

70. Zordan A (2011) Fluorescence in situ hybridization on formalinfixed, paraffin-embedded tissue sections. Methods Mol Biol 730: 189-202

71. Loken MR et al (2012) Residual disease detected by multidimensional flow cytometry signifies high relapse risk in patients with de novo acute myeloid leukemia: a report from Children's Oncology Group. Blood 120(8):1581-1588

72. Bacher $U$ et al (2009) Molecular diagnostics in acute leukemias. Clin Chem Lab Med 47(11):1333-1341

73. King RL et al (2011) A comparative analysis of molecular genetic and conventional cytogenetic detection of diagnostically important translocations in more than 400 cases of acute leukemia, highlighting the frequency of false-negative conventional cytogenetics. Am J Clin Pathol 135(6):921-928

74. Laforet MP et al (2013) Design and feasibility of a novel, rapid, and simple fluorescence 26-plex rt-PCR assay for simultaneous detection of 24 fusion transcripts in adult acute myeloid leukemia. J Mol Diagn 15(2):186-195

75. Ye F, Laosinchai-Wolf W, Labourier E (2012) An optimized technology platform for the rapid multiplex molecular analysis of genetic alterations associated with leukemia. Cancer Genet 205(10): 488-500

76. Marcucci G et al (2001) Quantification of CBFbeta/MYH11 fusion transcript by real time RT-PCR in patients with INV(16) acute myeloid leukemia. Leukemia 15(7):1072-1080

77. Basecke $\mathrm{J}$ et al (2002) Transcription of AML1/ETO in bone marrow and cord blood of individuals without acute myelogenous leukemia. Blood 100(6):2267-2268

78. Kusec R et al (1994) AML1/ETO fusion mRNA can be detected in remission blood samples of all patients with $\mathrm{t}(8 ; 21)$ acute myeloid leukemia after chemotherapy or autologous bone marrow transplantation. Leukemia 8(5):735-739

79. Miyamoto T, Weissman IL, Akashi K (2000) AML1/ETOexpressing nonleukemic stem cells in acute myelogenous leukemia with 8;21 chromosomal translocation. Proc Natl Acad Sci U S A 97(13):7521-7526

80. Wiemels JL et al (2002) In utero origin of $t(8 ; 21)$ AML1-ETO translocations in childhood acute myeloid leukemia. Blood 99(10):3801-3805

81. Falini B et al (2005) Cytoplasmic nucleophosmin in acute myelogenous leukemia with a normal karyotype. N Engl J Med 352(3):254-266

82. Colombo E et al (2005) Nucleophosmin is required for DNA integrity and p19Arf protein stability. Mol Cell Biol 25(20): 8874-8886

83. Colombo E et al (2002) Nucleophosmin regulates the stability and transcriptional activity of p53. Nat Cell Biol 4(7):529-533

84. Lindstrom MS (2011) NPM1/B23: a multifunctional chaperone in ribosome biogenesis and chromatin remodeling. Biochem Res Int 2011:195209

85. Falini B et al (2006) Immunohistochemistry predicts nucleophosmin (NPM) mutations in acute myeloid leukemia. Blood 108(6):1999-2005 
86. Pasqualucci L et al (2006) Mutated nucleophosmin detects clonal multilineage involvement in acute myeloid leukemia: impact on WHO classification. Blood 108(13):4146-4155

87. Schnittger $\mathrm{S}$ et al (2005) Nucleophosmin gene mutations are predictors of favorable prognosis in acute myelogenous leukemia with a normal karyotype. Blood 106(12):3733-3739

88. Cazzaniga G et al (2005) Nucleophosmin mutations in childhood acute myelogenous leukemia with normal karyotype. Blood 106(4):1419-1422

89. Schlenk RF et al (2008) Mutations and treatment outcome in cytogenetically normal acute myeloid leukemia. N Engl J Med 358(18):1909-1918

90. Gale RE et al (2008) The impact of FLT3 internal tandem duplication mutant level, number, size, and interaction with NPM1 mutations in a large cohort of young adult patients with acute myeloid leukemia. Blood 111(5):2776-2784

91. Brown $P$ et al (2007) The incidence and clinical significance of nucleophosmin mutations in childhood AML. Blood 110(3):979985

92. Yeamans $\mathrm{C}$ et al (2007) C/EBPalpha binds and activates the PU.1 distal enhancer to induce monocyte lineage commitment. Blood 110(9):3136-3142

93. Fos J et al (2011) Deficient CEBPA DNA binding function in normal karyotype AML patients is associated with favorable prognosis. Blood 117(18):4881-4884

94. Dufour A et al (2010) Acute myeloid leukemia with biallelic CEBPA gene mutations and normal karyotype represents a distinct genetic entity associated with a favorable clinical outcome. J Clin Oncol 28(4):570-577

95. Paz-Priel I, Friedman A (2011) C/EBPalpha dysregulation in AML and ALL. Crit Rev Oncog 16(1-2):93-102

96. Matsuo $\mathrm{H}$ et al (2014) Prognostic implications of CEBPA mutations in pediatric acute myeloid leukemia: a report from the Japanese Pediatric Leukemia/Lymphoma Study Group. Blood Cancer J 4:e226

97. Meshinchi S et al (2006) Clinical implications of FLT3 mutations in pediatric AML. Blood 108(12):3654-3661

98. Schnittger S et al (2002) Analysis of FLT3 length mutations in 1003 patients with acute myeloid leukemia: correlation to cytogenetics, FAB subtype, and prognosis in the AMLCG study and usefulness as a marker for the detection of minimal residual disease. Blood 100(1):59-66

99. Yamamoto Y et al (2001) Activating mutation of D835 within the activation loop of FLT3 in human hematologic malignancies. Blood 97(8):2434-2439

100. Kiyoi $\mathrm{H}$ et al (1998) Internal tandem duplication of the FLT3 gene is a novel modality of elongation mutation which causes constitutive activation of the product. Leukemia 12(9):1333-1337

101. Kottaridis PD et al (2001) The presence of a FLT3 internal tandem duplication in patients with acute myeloid leukemia (AML) adds important prognostic information to cytogenetic risk group and response to the first cycle of chemotherapy: analysis of 854 patients from the United Kingdom Medical Research Council AML 10 and 12 trials. Blood 98(6):1752-1759

102. Thiede $\mathrm{C}$ et al (2006) Prevalence and prognostic impact of NPM1 mutations in 1485 adult patients with acute myeloid leukemia (AML). Blood 107(10):4011-4020

103. Mead AJ et al (2008) Conflicting data on the prognostic significance of FLT3/TKD mutations in acute myeloid leukemia might be related to the incidence of biallelic disease. Blood 112(2):444 445, author reply 445

104. Mead AJ et al (2007) FLT3 tyrosine kinase domain mutations are biologically distinct from and have a significantly more favorable prognosis than FLT3 internal tandem duplications in patients with acute myeloid leukemia. Blood 110(4):1262-1270
105. Kottaridis PD et al (2002) Studies of FLT3 mutations in paired presentation and relapse samples from patients with acute myeloid leukemia: implications for the role of FLT3 mutations in leukemogenesis, minimal residual disease detection, and possible therapy with FLT3 inhibitors. Blood 100(7):2393-2398

106. Figueroa ME et al (2010) DNA methylation signatures identify biologically distinct subtypes in acute myeloid leukemia. Cancer Cell 17(1):13-27

107. Valk PJ et al (2004) Prognostically useful gene-expression profiles in acute myeloid leukemia. N Engl J Med 350(16):1617-1628

108. Paschka $\mathrm{P}$ et al (2010) IDH1 and IDH2 mutations are frequent genetic alterations in acute myeloid leukemia and confer adverse prognosis in cytogenetically normal acute myeloid leukemia with NPM1 mutation without FLT3 internal tandem duplication. J Clin Oncol 28(22):3636-3643

109. Figueroa ME et al (2010) Leukemic IDH1 and IDH2 mutations result in a hypermethylation phenotype, disrupt TET2 function, and impair hematopoietic differentiation. Cancer Cell 18(6):553567

110. Ho PA et al (2011) Leukemic mutations in the methylationassociated genes DNMT3A and IDH2 are rare events in pediatric AML: a report from the Children's Oncology Group. Pediatr Blood Cancer 57(2):204-209

111. Liang DC et al (2013) Cooperating gene mutations in childhood acute myeloid leukemia with special reference on mutations of ASXL1, TET2, IDH1, IDH2, and DNMT3A. Blood 121(15): 2988-2995

112. Damm F et al (2011) Prevalence and prognostic value of IDH1 and IDH2 mutations in childhood AML: a study of the AML-BFM and DCOG study groups. Leukemia 25(11):1704-1710

113. Markova J et al (2012) Prognostic impact of DNMT3A mutations in patients with intermediate cytogenetic risk profile acute myeloid leukemia. Eur J Haematol 88(2):128-135

114. Ribeiro AF et al (2012) Mutant DNMT3A: a marker of poor prognosis in acute myeloid leukemia. Blood 119(24):5824-5831

115. Delhommeau F et al (2009) Mutation in TET2 in myeloid cancers. N Engl J Med 360(22):2289-2301

116. Gaidzik VI (2012) TET2 mutations in acute myeloid leukemia (AML): results from a comprehensive genetic and clinical analysis of the AML study group. J Clin Oncol 30(12):1350-1357

117. Smith CC et al (2012) Validation of ITD mutations in FLT3 as a therapeutic target in human acute myeloid leukaemia. Nature 485(7397):260-263

118. Wander SA, Levis MJ, Fathi AT (2014) The evolving role of FLT3 inhibitors in acute myeloid leukemia: quizartinib and beyond. Ther Adv Hematol 5(3):65-77

119. Russler-Germain DA et al (2014) The R882H DNMT3A mutation associated with AML dominantly inhibits wild-type DNMT3A by blocking its ability to form active tetramers. Cancer Cell 25(4): 442-454

120. Dang L et al (2010) Cancer-associated IDH1 mutations produce 2hydroxyglutarate. Nature 465(7300):966

121. Ward PS et al (2010) The common feature of leukemia-associated IDH1 and IDH2 mutations is a neomorphic enzyme activity converting alpha-ketoglutarate to 2-hydroxyglutarate. Cancer Cell 17(3):225-234

122. Kandoth $\mathrm{C}$ et al (2013) Mutational landscape and significance across 12 major cancer types. Nature 502(7471):333-339

123. Ottone T et al (2008) An allele-specific rt-PCR assay to detect type A mutation of the nucleophosmin-1 gene in acute myeloid leukemia. J Mol Diagn 10(3):212-216

124. Chang TL et al (2003) Simplified capillary electrophoresis detection of the Flt-3 internal tandem duplications and D835 point mutations in acute myeloid leukemia. Haematologica 88(2): ELT04 
125. Murphy KM et al (2003) Detection of FLT3 internal tandem duplication and D835 mutations by a multiplex polymerase chain reaction and capillary electrophoresis assay. J Mol Diagn 5(2): 96-102

126. White BS, DiPersio JF (2014) Genomic tools in acute myeloid leukemia: from the bench to the bedside. Cancer 120(8):1134 1144

127. Alvarez S et al (2010) DNA methylation profiles and their relationship with cytogenetic status in adult acute myeloid leukemia. PLoS One 5(8):e12197

128. Bullinger L et al (2010) Quantitative DNA methylation predicts survival in adult acute myeloid leukemia. Blood 115(3):636-642

129. Zanotti L et al (2014) Diagnostic tests based on gene expression profile in breast cancer: from background to clinical use. Tumour Biol 35(9):8461-8470
130. Srivastava G et al (2014) Prospective multicenter study of the impact of oncotype DX colon cancer assay results on treatment recommendations in stage II colon cancer patients. Oncologist 19(5):492-497

131. Chatterton $Z$ et al (2014) Validation of DNA methylation biomarkers for diagnosis of acute lymphoblastic leukemia. Clin Chem 60(7):995-1003

132. Wertheim GB et al (2014) Microsphere-based multiplex analysis of DNA methylation in acute myeloid leukemia. J Mol Diagn 16(2):207-215

133. Rubnitz JE, Inaba H (2012) Childhood acute myeloid leukaemia. Br J Haematol 159(3):259-276

134. Schuback HL, Arceci RJ, Meshinchi S (2013) Somatic characterization of pediatric acute myeloid leukemia using next-generation sequencing. Semin Hematol 50(4):325-332 